\title{
Carnets
}

Revue électronique d'études françaises de l'APEF

Deuxième série - 20 | 2020

Imaginaire(s) du Voyage

\section{Victor Segalen, une conscience esthétique voyageuse}

\section{Adel Habbassi}

\section{(2) OpenEdition}

1 Journals

Édition électronique

URL : http://journals.openedition.org/carnets/12326

DOI : 10.4000/carnets. 12326

ISSN : 1646-7698

Éditeur

APEF

Référence électronique

Adel Habbassi, «Victor Segalen, une conscience esthétique voyageuse », Carnets [En ligne], Deuxième série - 20 | 2020, mis en ligne le 30 novembre 2020, consulté le 20 avril 2021. URL : http:// journals.openedition.org/carnets/12326 ; DOI : https://doi.org/10.4000/carnets.12326

Ce document a été généré automatiquement le 20 avril 2021.

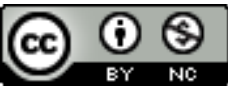

Carnets est mis à disposition selon les termes de la licence Creative Commons - Atribution - Pas d'utilisation commerciale 4.0 International. 


\title{
Victor Segalen, une conscience esthétique voyageuse
}

\author{
Adel Habbassi
}

Il ne semble pas que la notion d'espace humain soit universelle ; elle est un produit culturel de la langue et de la parole. (...) La littérature qui joue avec les codes et conventions de la langue est le lieu de l'imaginaire de l'espace.

(Grassin, 2000 : III-IV).

1 De par sa brièveté et la profondeur de ses expériences, la vie de Victor Segalen (1878-1919) offre plusieurs points communs avec celles des grands artistes. Valérie Bucheli rappelle que "les spécialistes de son œuvre lui reconnaissent» un certain nombre de «qualités » encore importantes de nos jours : « elle est conforme (...) à une certaine esthétique du cercle avancé de la production littéraire, grâce à son inscription très raisonnée dans des dispositifs modernistes » (Bucheli, 2020 : 2). Même si l'intérêt qu'ils présentent embrasse une multitude de domaines et plusieurs disciplines ${ }^{1}$, ses écrits nous font, surtout, découvrir un poète très intéressé par la diversité des hommes et de leurs modes de vie. Il a eu la chance d'exercer en tant que médecin dans la Marine française ce qui lui a permis de cultiver sa passion pour les arts, les voyages et les cultures du monde. Ses connaissances en archéologie ont élargi les centres d'intérêt de l'homme et le champ d'intervention du savant. Les missions à l'étranger auxquelles il a pris part, dans le cadre de son travail, et ses séjours prolongés en Polynésie et en Chine lui permirent de concilier entre écriture et voyage. Sa production d'essayiste et d'écrivain a, ainsi, consacré les qualités d'une littérature essentiellement voyageuse. Ses œuvres ont largement eu le temps de s'imprégner de la culture extrême-orientale, celle de la Chine (impériale) en particulier. Des textes comme Peintures (Segalen, 1916), Stèles (Segalen, 1973), Équipée (Segalen, 1929) et Les Immémoriaux (Segalen, 1956) essaiment des couleurs, des musiques et des parfums puisés dans ces régions. Les traits de cette écriture du monde trahissent une fascination exercée par les mythes de l'autre et ses livres d'histoire(s). Le regard dynamique du voyageur impatient de découvrir ces 
contrées lointaines crée les conditions d'un brassage permanent des différentes facettes du vivant, celles des hommes, de leurs habitus et de leurs imaginaires.

\section{Du voyage entre réel et imaginaire}

2 En 1909, Segalen est animé par une envie de découvrir et d'étudier les peintures des anciens empires chinois, mais il est vite déçu par l'extrême rareté de ces œuvres. Au lieu de le décourager, l'absence de supports cristallise ses idées. La sensibilité du poète comblera alors, souvent, les observations lacunaires de l'essayiste. Au voyage (réel) en Chine vient s'ajouter un autre voyage, à mi-chemin entre fiction et réalité. Peintures est, ainsi, un essai qui s'est naturellement métamorphosé en une prose poétique limpide sur des œuvres artistiques recréées par le voyageur français. Intitulée "Peintures magiques », la première partie débute et se clôt avec une "Ronde des immortels": comme dans les autres morceaux, le poète accompagne son lecteur, l'interroge, aiguise son "regard" :

Et maintenant, que le décor soit solide ou non, que cette esplanade, (vous la voyez, losangique et blanche, portée sur sa colonnade) se révèle d'albâtre ou de jade, ou taillée dans les mots ou dans le rêve... trouvez-vous donc une grande importance? Une grande différence? Les Esprits soufflent et règnent partout où Il veut. Ceci est la Peinture des Esprits, des Génies, des Immortels. Tout ce qui est peint ici n'a de concret que sa complaisance d'être vu. Tout ceci daigne apparaitre. Mais sachez bien, d'un souffle, tout ceci peut disparaître. Ces vieux hommes, nous devinons ce qu'ils expriment: la glorification de la durée. Ce sont les vieux fils du TempsEmpereur. (Segalen, $1916: 12$ )

La chaîne des conjonctions ( ou») et le champ du possible, ouvert par les interrogations qui interpellent le lecteur, projettent le sujet de l'œuvre " dans le rêve ». La machine de l'écriture puise dans l'extraordinaire potentiel du « rêve ", démultiplie ses formes, étend ses horizons. Comme le dit Jean-Marie Grassin dans notre épigraphe, "la littérature qui joue avec les codes et conventions de la langue est le lieu de l'imaginaire de l'espace» ouvert dans ces «peintures». Le "Temps» mythique des « vieux hommes » et la profondeur de cette « durée » tiennent dans les « mots ». « Tout ce qui est peint ici » est le fruit d'une subtile entente : «le décor », que le poète donne l'impression de décrire avec ses formes géométriques, ses couleurs, ses matières et le monde « concret » qu'il est censé comprendre, n'existe que par sa capacité « d'être vu » (Segalen, $1916: 12$ ).

4 De par la variété des termes qui le composent, le lexique de la vue («voyez... être vu... apparaître... ») fonctionne comme un prélude à l'intervention des facultés de l'esprit : «la Peinture des Esprits, des Génies, des Immortels» (Segalen, 1916:12) est à lire comme une variante imaginaire de ce "souffle» poétique (onirique) qui traverse la peinture-texte. Une lecture " géocritique $»^{2}$ du texte permet de dire que la consistance plurielle de cet univers légendaire porte les traces d'une saisie « polysensorielle » du monde. Inspirée par la culture taoïste, l'écriture de Peintures va au-delà d'un compte rendu visuel des paysages et des hommes qui les peuplent. Nous parlons, ici, de "polysensorialité, parce que la suprématie du regard sur les autres formes de perception sensorielle n'est pas culturellement universelle, et que tous les sens sont importants et présents dans la perception de l'espace » (Doudet, $2008: 8$ ). En prenant la forme d'une mise en garde ironique lancée au lecteur, l'introduction de l'œuvre souligne cette insuffisance de l'acte de « voir » : « Vous êtes là : vous attendez, décidés 
peut être à m'écouter jusqu'au bout ; mais destinés ou non à bien voir, sans pudeur, à tout voir jusqu'au bout? " (Segalen, $1916: 1$ ). Après avoir essayé de « tout voir jusqu'au bout ", l'intuition du lecteur et les éclairs de son imagination le transportent au(x) monde(s) fantastique(s) des peintures réinventées par Segalen.

Le préfixe qui est à la base de la formation du terme "polysensorialité » est fondamental dans la dynamique qui préside à cette saisie poétique du monde. Le choix de la première personne du pluriel («nous devinons ce qu'ils expriment») n'est point fortuit: le "je" qui se mêle à cette multitude de "façons de voir" subit, de moins en moins, le diktat du visible et adhère à une perception plurielle d'un univers pictural dont le signe particulier est la «différence ». Dès lors, "nous devinons ce qu'ils expriment... » (Segalen, 1916:12) est une formule heureuse qui confond la matière visible et les formes imaginaires qu'elle stimule :

Ce que vous allez enfin voir est en revanche, irrévocablement, la Dernière des Peintures Magiques.

Et vous conviendrez de vous-mêmes qu'à la suite de celle-ci, il est impossible d'imaginer une de plus, - même par ordre du Souverain régnant. (Segalen, 1916:

74)

6 Les pouvoirs de cette magie et les limites, infranchissables, de cette imagination représentent les traits caractéristiques des tableaux fabuleux de Peintures. Mohamed Ridha Bouguerra parle même d'un univers "fantastique» qui "nous révèle les créatures de cauchemar qui hantent nos rêves et habitent l'arrière-monde " (Bouguerra, 2017 : 182). La profusion de formes, de mouvements et de couleurs qui anime ce cortège d' " Esprits », de "Génies » et d'" Immortels » nous suggère la richesse de l'intertexte qui nourrit cette prose poétique. Le même critique souligne ce trait de l'œuvre et y décèle une " esthétique de l'outrance et de la démesure si chère à l'art décadent dans lequel a baigné la jeunesse de Segalen, à la fin du $\mathrm{XIX}^{\mathrm{e}}$ siècle » (Bouguerra, 2017 : 183). Les métamorphoses permanentes et l'irrationnel caractéristiques de ce Théâtre fabuleux décuplent le pouvoir du «rêve » et libèrent l'imagination du voyageur (occidental) qui réinvente le pays qu'il traverse à travers la sublimation de ses peintures.

7 Y a-t-il des interactions entre le monde créé par l'écrivain et le monde réel où nous vivons? De quelle nature seraient-elles? Ces questions ne cessent d'intéresser les critiques littéraires et les poéticiens : «L'espace représenté en littérature est-il coupé de ce qui lui est extérieur (comme le défendent les structuralistes) ou alors interagit-il avec lui ?», s'interroge Bertrand Westphal (Westphal, 2011: 162). Ces interactions éventuelles pourraient être stimulées par les déplacements du sujet-écrivant et ses périples. L'exemple de Victor Segalen est, à ce titre, significatif. Sa passion du voyage est telle qu'elle confond, parfois, les paysages traversés par le grand marcheur et les images (idées) qu'il en avait auparavant. Nous retrouvons cette coprésence du réel et de l'imaginaire dans Équipée, un livre écrit entre 1915 et 1916, mais dont il conçut la matière détaillée pendant une mission archéologique en Chine, en 1914.

Dès les premières pages d'Équipée, la question des liens dialectiques entre « réel » et «imaginaire » est vite posée : «(...) l'imaginaire déchoit-il ou se renforce quand il se confronte au réel ?», s'interroge l'auteur (Segalen, 1929: 11). Il est ainsi clair que le voyage contribue à la cristallisation de cette confrontation. En dépit de leurs natures antagoniques, ces deux catégories perceptives aiguisent, en permanence, notre manière de sentir le monde (in)visible, les êtres et les choses. Que dire alors si cette capacité de sentir s'applique aux "vérités" du corps, à celles de l'esprit et aux 
métamorphoses du (des) monde(s) de nos rêves? La coexistence du réel et de l'imaginaire chez l'homme est, en effet, révélatrice de la complexité de son rapport au monde et aux autres. Et l'archéologue d'Équipée en est parfaitement conscient. Il sait également que les dynamiques spatio-temporelles qui travaillent les sens du voyageur sont, à ce propos, fort intéressantes: Segalen en a développé l'expérience avec une précision quasi scientifique. Le lecteur est également prévenu : un voyage de ce type ne s'improvise pas. Avant de s'y engager, on rêve les lieux où on se déplacera, on imagine les hommes, la faune, la flore et les paysages qu'on y trouvera. Et il est étonnant que l'auteur d'Équipée le dise avec des termes empruntés à certaines formes d'expression artistique et au lexique (de l'explication) des rêves. Il est, alors, question de "se dessiner le voyage » (Segalen, $1929:$ 14), de « la mise en scène du voyage » (Ibid., : 12), de « voyage double » ou de « double voyage » (Segalen, 1929: 50). Épuisé et de moins en moins lucide, le marcheur "compose [son voyage] entre la courbature et l'appétit grandissant » (Segalen, 1929 : 24) ou bien s'arrête parce qu'il est « pris d'un doute plus fort que tous les autres, pris tout d'un coup du vertige et de l'angoisse du réel» (Segalen, 1929 : 19). Mais, encore une fois, les paysages qui défilent dissipent la fatigue et mêlent le monde "réel » aux productions imaginaires d'un esprit qui a du mal à en saisir la grandeur. Nous en trouvons un très bel exemple dans le septième chapitre du même livre, intitulé "La montagne à gravir»: «Mais j'imaginais tout autre la domination divine de la montagne» (Segalen, 1929: 29), s'exclame Segalen devant l'extrême grandeur et l'élévation des chaînes montagneuses dans cette partie de l'Asie. L'intervention de l'imagination réfère, entre autres, un « tout autre » regard porté sur le gigantisme de cette nature qui domine l'homme, lui rappelle sa fragilité, approfondit sa méditation. Ce faisant, le visiteur occidental prend conscience des limites de sa connaissance du monde et (de la force) des hommes qui le peuplent.

Ce qui est esquissé dans Équipée sera étayé et développé dans Notes sur l'Exotisme (Segalen, 1955) et Essai sur l'exotisme ${ }^{3}$. Souvent prévus, au départ, comme des journaux de bord chaotiques ${ }^{4}$ des lieux (réels et imaginaires) traversés par l'artiste, ces textes se sont développés pour parler de l'âme profonde des peuples et de leurs cultures (ancestrales). Au fur et à mesure de ses longs voyages, Segalen a commencé à mettre en œuvre une esthétique adaptée à sa manière d'aller vers l'autre, de saisir ses légendes et d'explorer sa mémoire collective. Autant d'informations et de remarques soigneusement enregistrées dans les carnets qui ont fourni la documentation de ses périples : nous y trouvons sa conception de « l'exotisme » et l'intelligence culturelle qui la nourrit, sources intarissables de ses récits (mythiques) et de ses poésies. Les membres de sa famille ont vite saisi l'importance de ces documents et en ont publié une bonne partie dans Notes sur l'exotisme.

\section{Un exotisme soumis au prisme du Divers}

10 Essai sur l'exotisme est un autre texte posthume dans lequel Segalen revisite la notion d'«exotisme» en lui redonnant une certaine originalité. Les lecteurs, gavés des divagations de Bernardin de Saint-Pierre et bercés par la couleur locale (indigène) reproduite par Pierre Loti, découvriront dans cette autre dimension de l'« exotisme ", purgée de l'emprise coloniale et de l'attitude européocentriste qui en découle, un cheminement et une sorte d'ascèse qui les initient à cet art de la différence caractéristique de la poétique de Victor Segalen. Les accents nouveaux de cette prose 
fluide du monde rompent avec la monotonie des cadastres ethnographiques écrits sous le contrôle des armées coloniales. Bien entendu, c'est aux artistes qu'incombe le devoir d'assumer le « combat » éthique impliqué par cette « esthétique du Divers »; les poètes $\mathrm{y}$ seront en première ligne :

Le Divers décroît. Là est le grand danger terrestre. C'est donc contre cette déchéance qu'il faut lutter, se battre, mourir peut-être avec beauté. Les poètes, les visionnaires mènent toujours ce combat, soit au profond d'eux-mêmes, soit - et je le propose - contre les murs de la Connaissance. (Segalen, 1978 : 95)

11 Parmi les causes qui sont à l'origine de ce « grand danger terrestre » (Segalen, 1978 : 95) et de cette "déchéance ", certaines niaient, encore au début du XXème siècle, la diversité de l'humain et ses trésors imaginaires : racisme, xénophobie, nationalismes exacerbés... ont longtemps réduit l'histoire, le savoir et le progrès aux limites géographiques et épistémologiques de l'Occident. Cet autisme culturel et cette négation de l'autre ont ouvert la voie à un universalisme colonial dont les conséquences minent, encore aujourd'hui, nos représentations de l'autre et de ses traditions. Christian Doumet souligne l'importance du contexte mondial dans lequel Segalen avait conçu son "esthétique du Divers»: «comment oublier qu'au moment où paraissent Les Immémoriaux, la colonisation française connaît son apogée? » (Doumet, 1983 : 91). La mainmise du colon sur la culture de l'autre et le dénigrement de ses symboles (sacrés) produisent l'image d'un "indigène" dont le spectacle folklorique est exotique. Les préjugés raciaux et culturels sur la base desquels le colon méprise ces populations d'outre-mer naissent de cette vision déformée (et déformante) de l'autre.

Ceci est d'autant plus vrai que Segalen envisage la possibilité d'une fascination par le "divers" selon un "parallélisme entre le recul dans le passé (Historicisme) et le lointain dans l'espace (Exotisme)» (Segalen, 1955 : 385). L'auteur de Notes sur l'Exotisme tient absolument à dépouiller son "esthétique du Divers" de tous ces malentendus. Bien avant Aimé Césaire, le jeune poète breton n'oublie pas, lui aussi, que la "beauté » de son "combat" tient aux " armes miraculeuses", expression que nous empruntons au livre d'Aimé Césaire (1946), dont il dispose, celles de la culture et de la " connaissance » authentique de l'autre en particulier. L'auteur des Immémoriaux le fait lorsqu'il nous guide dans une découverte des spécificités et des symboles de la culture maorie. Le lecteur finit par comprendre que la profusion imaginaire qui est à la base de ces héritages millénaires confond les visées ethnographiques et leur exotisme de pacotille. La valorisation des héritages culturels de ces peuples, vivant à des milliers de kilomètres de l'Europe, peut être perçue comme une réponse à la politique hégémonique propre à toute stratégie coloniale. Une fois occupé, le territoire est livré aux missionnaires: Segalen remarque, avec beaucoup d'ironie, que leur œuvre civilisatrice dans les Iles Marquises menace de corrompre l'innocence des tribus maories et les pousse même à s'entretuer.

À une époque où la logique colonialiste consacrait la domination militaire de l'Occident sur plusieurs régions du monde, un jeune poète français récusait, donc, la portée réductrice de cette vision exotique de l'autre. Fidèle aux enseignements de sa culture de grand voyageur, il a choisi de modifier les connotations du terme « exotisme ", alors qu'il pouvait le rejeter. Dans Essai sur l'exotisme, il cerne les nuances d'un mot débarrassé de ses significations superficielles. Gilles Manceron explique que l'auteur de cet essai s'était servi du mot " exotisme (...) en opérant un véritable détournement de sens puisqu'il l'a utilisé pour décrire une problématique diamétralement opposée " (Manceron, 1991 : 14). Pour s'entendre sur cette acception autre du mot, Victor Segalen 
pose une condition préalable, essentielle, « le dépouiller de ses oripeaux : le palmier et le chameau; casque de colonial; peaux noires et soleil jaune " (Segalen, 1978: 41). L'exploration lucide de la (des) culture(s) et de la (des) population(s) de chaque territoire l'emporte sur l'attitude touristique passive et béate : «l'exotisme n'est donc pas cet état kaléidoscopique du touriste et du médiocre spectateur, mais la réaction vive et curieuse au choc d'une individualité forte contre une objectivité dont elle perçoit et déguste la distance » (Segalen, 1978: 43). " La sensation d'Exotisme » naît, ainsi, du « choc » et de la confrontation de différences non apprivoisées.

Dans le même livre, Segalen s'attarde sur cette «sensation d'Exotisme » en précisant qu'elle «n'est autre que la notion du différent; la perception du Divers; la connaissance que quelque chose n'est pas soi-même; et le pouvoir de l'exotisme, qui n'est que le pouvoir de Concevoir autre" (Segalen, 1978: 41). Alerte, la pensée du voyageur progresse à travers une série de définitions et de synonymes qui interfèrent : «l'Exotisme », « le différent ", « le Divers ». L'expérience de l'altérité est décisive pour se doter du "pouvoir de l'exotisme», tandis que «la perception du Divers» est inséparable du "pouvoir de Concevoir autre». Segalen a même forgé un mot pour désigner celui qui a cette "individualité forte » et qui ne se contente pas de subir le "Divers », mais « le pénètre, l'assaille, le réveille et le trouble ", il s'agit de "l'Exote » (Segalen, $1978: 40)^{5}$. Est « Exote » celui (ou celle) qui sait garder une certaine distance avec la différence qui l'interpelle. Cela est proche d'un état d'inassouvissement volontaire: "Ne nous flattons pas d'assimiler les mœurs, les races, les nations, les autres; mais au contraire réjouissons-nous de ne le pouvoir jamais, nous réservant ainsi la perdurabilité du plaisir de sentir le Divers"(Segalen, 1978: 44). Les conséquences du décentrement du voyageur européen sont notables. Il n'est plus question " d'intégrer dans une vision du monde des éléments de décor venus d'outremer, mais de considérer les autres civilisations en elles-mêmes, sans se contenter de les évaluer à la toise des critères occidentaux » (Manceron, 1991 : 14). Nous sommes aux antipodes des visées assimilationnistes de la machine coloniale qui ne cherchait qu'à uniformiser pour dominer et exploiter.

\section{Le Divers, un motif éthico-esthétique}

15 Le sous-titre d'Essai sur l'exotisme (Une esthétique du Divers) souligne que le « Divers » est également un motif artistique : "Mais pour moi, c'est une aptitude de ma sensibilité, l'aptitude à sentir le divers, que j'érige en principe esthétique de ma connaissance du monde », affirme l'essayiste (Segalen, $1978: 50$ ). Attentive aux appels du "divers", cette pensée erratique est illuminée des couleurs du monde. Le projet « esthétique » qui se précise aspire même à une poétique du «Divers". En service à bord de l'aviso La Durance (1903-1905), le jeune médecin commence à cultiver cette «aptitude » de sa "sensibilité ». Sur ses carnets de voyageur, il s'applique à commenter les traits spécifiques des terres et des hommes qu'il découvre à travers le Pacifique. Les textes qui naîtront de ces rencontres sont habités par des mémoires millénaires. Les Immémoriaux raconte la disparition d'une parole porteuse des origines maories. Segalen s'est longuement penché sur les cultures de ses hôtes pour en déchiffrer les traces. Loin de mettre un terme à l'aventure du "Divers ", la mort des derniers détenteurs de cette parole relance la quête de leurs origines. En apprenant la langue de l'autre, le jeune Breton accède aux codes d'une culture extrême-orientale mythique. La méditation sur 
les Stèles lui permet de tisser des métaphores poétiques propices à la résurrection d'un imaginaire et d'une scénographie très proches des dynasties de la Chine impériale.

Cette écriture du voyage, soucieuse de son image et de ce qu'elle réfléchit des autres, est filigranée dans les signes du texte. La typographie de Stèles donne à voir, et à penser, un «Divers" qui stimule l'acte de lecture. Les idéogrammes chinois se mêlent aux phrases écrites en français pour mettre en valeur cette altérité absolue. Telle une forêt de signes hétérogènes, la page consacre "la notion du différent» qui nourrit ce «Divers ». Cette façon de sentir la différence (de l'autre) ouvre, au sein du langage, un espace où se manifestera, progressivement et des deux côtés, une identité en devenir. Segalen vit pleinement ce face-à-face avec une altérité qu'il se garde de percevoir avec des normes occidentales. Certes, le mur dressé par le "Divers " pourrait lui renvoyer quelques reflets de sa propre culture, mais l'essentiel de cette perception de l'autre réside, avant tout, dans le vertige du décentrement. Parti sur les traces d'« une vierge occidentale ", le poète rencontre une autre vierge, orientale, "Kiang-yuan, fille sans défaut (qui) devint mère" (Segalen, 1973: 14). "Éloge d'une vierge occidentale" débute et se clôt avec le même vers : «la raison ne s'offense pas : certainement une vierge occidentale a conçu » " (Segalen, 1973 : 14). Le terme "raison » est crucial pour la symbolique de cette rencontre entre Occident et Extrême-Orient. Le culte de "la raison occidentale" se réfléchit dans les bifurcations de la magie orientale. Assez différent, le sacré de ces contrées éloignées de l'Europe a été « conçu » dans un espacetemps antérieur à la généalogie et à la "logique" monothéistes.

Si Abdelkébir Khatibi (1938-2009) a été séduit par la pensée de Victor Segalen, c'est qu'il était, lui-même, un arpenteur du monde dont il a longuement décrypté les cultures. Sociologue franco-marocain de formation, il a développé ses visions philosophique et esthétique dans plusieurs textes où l'essayiste échange, en permanence, avec l'écrivain. Le nom de Khatibi est souvent associé à celui de Segalen depuis sa participation, en 1984, à un congrès mondial sur les littératures de langue française. "Célébration de l'exote » est un article dans lequel le penseur marocain rend hommage à une «identité en devenir dans un nouvel internationalisme littéraire " (Khatibi, $1984: 365)$. L'auteur de Figures de l'étranger dans la littérature française analyse le rapport dialectique qui s'est créé entre les idées de Segalen et ce système (clos) qu'était la littérature française : "Segalen se soumet à l'exigence du dehors, il en fait une loi d'écriture. Il ne peut écrire que sur ce dehors, sur les étrangers. Dehors du territoire et marges du livre français, dehors des valeurs de l'écrivant, de son système de référence" (Khatibi, 1987: 15). Nous apprenons que l' "esthétique du Divers » de l'écrivain français est rythmée par une dynamique essentiellement centrifuge. Le "système de référence" et ses codes partent en éclats, le « territoire » du langage s'étend et dissémine les signes culturels de «l'écrivant ». La pensée du "dehors » déborde les limites du système, donne sur les périphéries, brouille efficacement les configurations d'un monde longtemps soumis aux lois du centre.

La rupture qui s'est opérée entre l'exotisme pittoresque ${ }^{6}$ et «l'exotisme » segalenien tient également à un mouvement diachronique, à l'œuvre dans la "perception du Divers». Parce que «l'exotisme n'est pas seulement donné dans l'espace, mais également en fonction du temps» (Segalen, 1978: 41). La distance créée par (dans) le passé fonctionne tel un paradigme générateur de la différence irréductible à laquelle se confronte "l'Exote ». Les cultures et les mondes dans lesquels se sont réalisés les rites maoris et les stèles chinoises produisent un « choc » inhérent à la rencontre avec cette 
différence sauvage. Khatibi parlera, à ce propos, d'une "différence intraitable " (Khatibi, 1987 : 20). L'abîme ouvert par la perspective historique concrétise, sur l'axe du temps, la « distance » qui fait l'authenticité de la « sensation du Divers ». Nous relevons cet impact du temps dans les affinités qu'il a avec le terme «éternelle » dans une autre définition de "l'exotisme » proposée par Segalen: "L'exotisme n'est donc pas une adaptation (...), mais la perception aigüe et immédiate d'une incompréhensibilité éternelle" (Segalen, 1978: 44). "Le pouvoir de Concevoir autre", condition fondamentale du surgissement du "Divers", serait anéanti par une éventuelle familiarisation du sujet avec les images de l'autre. Le maintien d'un état d'inadaptation, dérangeante, intensifie le travail du rêve : le manque engendré par les zones d'ombre de l'inconnu renouvelle le désir de prolonger le face-à-face avec une altérité indomptable. En tant que trame de cette "esthétique du Divers", l'imaginaire est stimulé par les possibilités du rêve qui est, par excellence, l'espace-temps de l'indéfini et de l'imprécis. Ainsi conjugués, l'imaginaire et le rêve surdéterminent les visions du voyageur et ses productions mentales.

19 Cinquante ans après Segalen, Édouard Glissant reprendra le terme «Divers " pour l'adapter à une pensée poétique et philosophique proche de la réalité antillaise. L'auteur de L'Intention poétique (Glissant, 1969) se méfie de la notion d'« universel » telle qu'elle a toujours prévalu dans l'histoire et la culture occidentales. Les voyages (forcés) dont il évoque les itinéraires historiques et imaginaires ont déterminé la réalité géoculturelle complexe de sa terre natale. La manière dont l'Archipel de la Caraïbe s'est constitué, démographiquement et culturellement, à travers les siècles servira d'exemple de ce qu'il appelle une «créolisation» progressive du monde ${ }^{7}$. Sur le sol antillais, les colons partis des côtes européennes précipiteront ce croisement, parfois douloureux, de traditions et d'hommes très divers ${ }^{8}$. La mise en relation des langues et des mœurs d'autant de peuples fera que «le monde se créolise, c'est-à-dire que les cultures du monde mises en contact de manière foudroyante (...) se changent en s'échangeant à travers les heurts irrémissibles, des guerres sans pitié mais aussi des avancées de conscience et d'espoir » (Glissant, 1995 : 44).

20 La concentration, dans l'espace et dans le temps, d'une population amérindienne, de plusieurs vagues d'esclaves africains acheminés par les négriers et de milliers de migrants venus d'autres continents constituera, à travers les siècles, un «ToutMonde» (Glissant, 1997) dans lequel les groupes et les individus finiront par s'influencer. Dans cette ouverture de l'archipel, Glissant discerne le mouvement d'un «Diversel» (Glissant, 1997: 89) qui l'emporte sur l'universel européocentriste et sa logique coloniale. La densité culturelle de ce "Tout-Monde » puise dans les richesses des traditions et des imaginaires charriés par autant d'esclaves et de migrants. Les énergies qui sont à l'œuvre dans cet espace pluriethnique privilégient ce qui relie et mène vers (à) l'autre: "car la poétique de la Relation suppose qu'à chacun soit proposée la densité (l'opacité) de l'autre» (Glissant, 1969: 23). À l'instar de Victor Segalen, ce philosophe épris de voyages, de poésie et de roman développe sa conception de la création littéraire (et artistique) dans le cadre d'une "poétique du Divers" (Glissant, 1995). L'ancrage identitaire n'y est plus exclusif, puisque chaque identité s'enrichit et se métamorphose en (s') échangeant avec d'autres.

21 L'intérêt particulier porté par Segalen aux peintures de Paul Gauguin pourrait être interprété comme un prolongement du face-à-face avec ces identités et ces corps différents. Dans un hommage qu'il rend à ce peintre, la fascination par l'être maori est 
plus qu'évidente : «L'homme maori ne peut pas s'oublier quand on l'a vu, ni la femme cesser d'être aimée quand on l'aime. Paul Gauguin sut aimer là-bas, et voir plus puissamment que tout être... » (Joly-Segalen, $1950: 8$ ). Le retour insistant des verbes « voir » et « aimer » atteste les traces profondes de ces métamorphoses du corps et de l'esprit : la puissance du regard est à l'origine des scènes et des formes (in)conscientes qui se créent sur (dans) les toiles de l'artiste. L'univers pictural s'avère idéal pour la mise en scène de cette interpénétration du réel et de l'imaginaire. En impliquant « puissamment» l'esprit, le rêve et l'imagination, les gestes artistiques stimulés par les itinéraires et les rencontres réinventent le voyage, redéfinissent ses objectifs, varient ses créations. Si l'énergie dépensée lors de ce cheminement est inépuisable, c'est que la permanence du champ de l'inconnu intensifie le désir de ces rencontres. Dès lors, l'impossibilité d'«oublier » ces corps nous dit, autrement, l'impact de l'impression du sujet artistique. En se liant à une femme maorie ${ }^{9}$, le jeune voyageur, aurait-il tenté de vivre, de très près, ce "pouvoir de Concevoir autre " et cette mise en abyme de son identité dans un corps (absolument) autre?

\section{Pour conclure}

En 1906, Segalen a écrit un livre dans lequel il exprime sa fascination par «l'homme aux semelles de vent ». Le Double Rimbaud (Segalen, 1986) s'interroge également sur le mystère qui enveloppe les liens entre les voyages de l'aventurier et la quête d'un " ailleurs " assumée par le poète d'Une saison en enfer et Illuminations. Pour l'auteur de Notes sur l'Exotisme, l'énigme de cette coïncidence du silence de Rimbaud et de ses voyages interminables est totale ; lui qui a toujours conçu le voyage et les horizons qu'il ouvre comme un immense espace littéraire et un vivier de l'écriture. Aux voies ouvertes par Arthur Rimbaud s'ajoutent, au début du XXème siècle, celles que tracent la pensée poétique et les textes de Victor Segalen.

Après la deuxième Guerre mondiale, cette manière de découvrir l'autre au gré de voyages réels et imaginaires ne cesse d'inspirer beaucoup d'écrivains, des artistes et des poéticiens à travers le monde. Et c'est la "géopoétique » de Kenneth White qui nous vient à l'esprit. Rappelons que ce dernier a eu "l'idée » première de ce qu'il présente comme une « théorie » lors d'une traversée de l'Amérique du nord:

C'est en 1979, en voyageant, pérégrinant, déambulant le long de la côte nord du Saint-Laurent, en route vers le Labrador, que l'idée de la géopoétique a pris forme.

J'ai relaté ce voyage, j'ai essayé de dire toute l'ampleur de la sensation, de l'idée,

dans le livre La Route bleue. (White, 2008)

Les termes " côte ", " route ", " voyage » et cette passerelle " poétique » qui mêle la " sensation » de celui qui réfléchit en " déambulant » aux mots de son « livre " sont des traces de cette littérature voyageuse qui s'est renouvelée depuis les Notes méticuleuses de Segalen. Kenneth White ne cache pas cette influence et va jusqu'à voir en l'auteur d'Équipée un illustre prédécesseur. Il développe, d'ailleurs, cette réflexion dans un livre où il aborde les affinités du « lieu et [de] la formule » dans l'aventure rimbaldienne et dans les expériences esthétiques développées par cet illustre voyageur que fut Segalen (White, $2007: 28$ ).

Par ailleurs, dès les années 60 , cette culture du voyage, cette conception originale de "l'exotisme » et les idées qui en découlent bouleverseront les angles de vue et les méthodes qui prévalaient dans plusieurs études philosophiques, sociologiques et 
anthropologiques. Dans Civilisés, dit-on, Georges Balandier souligne l'importance du passage d'une ethnographie traditionnelle, réductrice des richesses de l'humain, à une anthropologie plus attentive aux réalités du monde contemporain : «L'anthropologie, en tant que mode de connaissance des cultures et des sociétés, a d'abord été l'exploratrice du divers (...). Ce faisant, la différence est placée au centre de ses interrogations " (Balandier, 2003 : 252-253). L'auteur distingue nettement l'attitude de l'ethnographe et celle de l'anthropologue. Mêlé aux expéditions coloniales, le premier sombrait souvent dans les illusions de "l'inégalité des races", alors que le deuxième essaie de rendre compte de la complexité du vivant en tentant « une réappropriation des différences" (Balandier, 2003: 255). Les résultats de cette dernière approche sont édifiants. Les horizons ouverts par la perspective dynamique empruntée par le voyageur débarrassé de ses préjugés (culturels) entament une réconciliation des peuples et des imaginaires du monde, «ce qui rend possible l'expression de l'humain dans le maintien de la diversité » (Balandier, 2003 : 255). Quant à Pierre Bouvier, il présente sa "socio-anthropologie » comme un terrain de recherche pluridisciplinaire qui « a pour tentative la densité existentielle de l'individuel et du collectif, du sujet et des altérités maintenues ou métissées " (Bouvier, 2000 : 11). Depuis Essai sur l'exotisme, les sciences humaines poursuivront cette démystification de la notion d'" altérité ». Les innombrables études menées sur les processus de construction identitaire, du sujet et de son groupe, montrent combien "je" ("nous") et "l'autre" ("les autres") sont inextricablement liés. Les conséquences de ces rapports complexes à l'autre sur nos manières de dire, de penser et de créer expliquent l'importance, plus que jamais actuelle, de l'« esthétique du Divers » mise au point par Victor Segalen.

\section{BIBLIOGRAPHIE}

BALANDIER, Georges (2003). Civilisés, dit-on. Paris : Presses Universitaires de France.

BOSSUET, Camille (le 1 août 2008). « Une esthétique du Divers ", article mis en ligne (le 1 août 2008) sur le blog La Plume francophone : les littératures du monde francophone [Disponible le 12 mars 2020]. URL https://la-plume-francophone.com/2008/08/01/victor-segalen-essai-sur-lexotisme/

BOUGUERRA, Mohamed Ridha (2016). "Écriture, réécriture et art pictural dans Peintures de Victor Segalen ", Littératures, $n^{\circ} 74$ : Pratiques et enjeux de la réécriture: L'écrivain au défi du peintre, pp. $175-185$.

BOUILLIER, Henry (1961). Victor Segalen. Paris : Mercure de France (Nouvelle édition revue et corrigée en 1986 : collection Ivoire).

BOUVIER, Pierre (2000). La Socio-anthropologie. Paris : Armand Colin.

BUCHELI, Valérie (2020). « Le cas Victor Segalen. À propos de deux mises à l'écart successives ", Contextes (revue de sociologie de la littérature), $\mathrm{n}^{\circ} 27$ (Autopsie de l'échec littéraire), mis en ligne : 2020 [Disponible le 02 octobre 2020). URL https://doi.org/10.4000/contextes.9112

BURGOS, Jean (1982). Pour une poétique de l'imaginaire. Paris : Seuil. 
CÉSAIRE, Aimé (1946). Les Armes miraculeuses. Paris : Gallimard.

CORDONIER, Noël (2017). «Victor Segalen, La Stèle », Médium, n 50 : « Littérature, chutes et rebonds ». Paris : Gallimard, pp. 76-89.

DOUDET, Caroline (2008). « Géocritique : théorie, méthodologie, pratique », Acta fabula, vol. 9, $\mathrm{n}^{\circ}$ 5, Mai 2008 [Disponible le 01 octobre 2020], URL : http://www.fabula.org/revue/ document4136.php

DOUMET, Christian (1983). «Écriture de l'exotisme : Les Immémoriaux de Victor Segalen », in Littérature, n 51, (Poésie), pp. 91-103.

GLISSANT, Édouard (1969). L'Intention poétique. Paris : Seuil.

GLISSANT, Édouard (1995). Introduction à une poétique du Divers. Paris : Gallimard.

GLISSANT, Édouard (1997). Traité du Tout-Monde. Paris : Gallimard.

GLISSANT, Édouard (2007). Mémoires des esclavages. Paris : Gallimard, nrf.

GRASSIN, Jean-Marie (2000). " Pour une science des espaces littéraires ", La Géocritique, mode d'emploi (sous la direction de Bertrand Westphal). Limoges : Presses Universitaires de Limoges (Introduction).

JOLY-SEGALEN, Annie éd. (1950). Lettres de Gauguin à Daniel de Monfreid. Précédées d'un hommage à Gauguin par Victor Segalen. Paris : Éditions Georges Falaize.

KHATIBI, Abdelkebir (1984). "Célébration de l'exote », in Giuliana Toso-Rodinis (éd.). Actes du Congrès mondial des littératures de langue française. Padoue : Université de Padoue, pp. 363-367.

KHATIBI, Abdelkebir (1987). Figures de l'étranger dans la littérature française. Paris : Denoël.

MANCERON, Gilles (1991). Segalen. Paris : éditions J-C. Lattès.

POSTEL, Philippe (2001). Victor Segalen et la statuaire chinoise. Paris : Honoré Champion.

Réunion des musées nationaux (France), Musée d'Orsay (Paris), Museum of fine arts (Boston, Mass.) (2004). Gauguin Tahiti : l'atelier des tropiques (Paris, Galeries nationales du Grand Palais, 30 septembre 2003-19 janvier 2004. Paris, Boston, Museum of fine arts, 29 février-20 juin 2004), Paris : Grand Palais, Réunion des musées nationaux.

SEGALEN, Victor (1916). Peintures. Paris : Georges Crès et $C^{\text {ie }}$.

SEGALEN, Victor (1929). Équipée. Paris : Gallimard.

SEGALEN, Victor (1955). Notes sur l'Exotisme. Paris : Mercure de France.

SEGALEN, Victor (1956). Les Immémoriaux. Paris : Plon.

SEGALEN, Victor (1973). Stèles. Paris : Gallimard, Collection Poésie.

SEGALEN, Victor (1978). Essai sur l'exotisme : Une esthétique du Divers. Paris : Fata Morgana.

SEGALEN, Victor (1986). Le Double Rimbaud. Paris : Fata Morgana, Bibliothèque artistique et littéraire.

SEGALEN, Victor (2011). Cuuvres complètes (Textes établis et annotés par Philippe Postel : en trois volumes). Paris : Honoré Champion.

WESTPHAL, Bertrand (2000). « Pour une approche géocritique des textes, esquisse », in La Géocritique, mode d'emploi. Limoges : Presses Universitaires de Limoges, pp. 9-40.

WESTPHAL, Bertrand (2011). La Géocritique. Réel, fiction, espace. Paris : Éditions de Minuit. 
WHITE, Kenneth (2007). Les Finisterres de l'Esprit - Rimbaud, Segalen et moi-même. Paris : Édit. Isolato. WHITE, Kenneth (2008). La Géopoétique : site officiel de Kenneth White [Disponible le 30 mars 2020] URL http://www.kennethwhite.org/geopoetique/.

\section{NOTES}

1. Voyage, histoire, géographie, anthropologie (culturelle), sociologie, linguistique, arts, littérature, etc. Cette multiplicité des horizons ouverts par l'œuvre nous renseigne aussi sur les passions de l'homme et sur les innombrables talents de l'artiste. C'est ainsi que le présente Camille Bossuet: «ce voyageur et médecin, musicien averti et linguiste, archéologue et calligraphe, fumeur d'opium, dessinateur, photographe, éditeur... est en effet d'abord reconnu comme sinologue, ethnologue... Et poète » (Bossuet, 2008, s.p.).

2. Pour élucider ce concept et les thèses qui le développent, Bertrand Westphal présente les «quatre points de l'approche géocritique: la multifocalisation, la polysensorialité, la stratigraphie et l'intertextualité » (Westphal, 2011 : 200).

3. Il s'agit d'une série de notes rédigées par Segalen durant ses voyages et publiées, en 1978, après sa mort, sous le titre Essai sur l'exotisme : Une esthétique du Divers. Paris : Fata Morgana.

4. Les carnets de voyage qui ont fourni la matière de ces ouvrages consistaient en une multitude de remarques, témoignages et impressions (sans fil conducteur) sur les contrées et les populations que Segalen découvrait à chaque étape de ses périples. Dans la plupart de ses livres, l'auteur réécrivait cette matière abondante en mettant de l'ordre (chronologique, thématique, scientifique, etc.) dans toutes ces notes.

5. Dans la même page, Segalen précise : «L'attitude ne pourra pas donc être le je qui ressent mais au contraire l'apostrophe du milieu au voyageur (...). C'est le tu qui dominera ». (Segalen, 1978 :

40)

6. Celui du voyageur attiré par la couleur locale et les tableaux folkloriques.

7. Glissant précise que «le mot créolisation vient du terme créole et de la réalité du monde créole » (Glissant, $1995:$ :18).

8. «La néo-América et l'archipel caraïbe préfigurent des relations d'un nouveau type, qui se sont ensuite établies dans le monde. C'est ce que nous voulons dire quand nous affirmons que le monde se créolise. Il ne devient pas créole, il entre dans ses diversités » (Glissant, 2007 : 107-108).

9. Il s'agit de Maraa Vahine, l'épouse maorie de Victor Segalen. Sa photographie est collée sur le journal personnel de l'écrivain. Ces informations ont été recueillies dans le catalogue de l'exposition qui a eu lieu aux Galeries nationales du Grand Palais (Paris) et au Museum of fine arts (Boston) entre 2003 et 2004 : Réunion des musées nationaux (France), Musée d'Orsay (Paris), Museum of fine arts (Boston, Mass.) (2004). Gauguin Tahiti : l'atelier des tropiques. Paris: Grand Palais, Réunion des musées nationaux, p. 324.

\section{RÉSUMÉS}

Médecin de formation, Victor Segalen avait bien d'autres talents. Engagé dans la Marine française, il s'est rendu en Polynésie et en Chine, allant à la rencontre d'autres civilisations. Les carnets de notes qu'il remplira lors de ces voyages fourniront la matière principale de ses écrits 
ultérieurs. Alors que les empires coloniaux faisaient mainmise sur les richesses des peuples d'Afrique et d'Asie, ce poète épris de la diversité du monde refuse la vision folklorique de l'autre ; il récupère le mot " exotisme » et l'inscrit dans " une problématique diamétralement opposée " (Manceron, 1991 : 14). L'« esthétique du Divers », exposée dans son Essai sur l'exotisme, accorde un rôle prépondérant à "l'imaginaire " et aux possibilités du "rêve " comme "aptitudes » fondamentales de «l'Exote » : un type de voyageur doté de ce "pouvoir de Concevoir autre ». La richesse de cette façon de percevoir l'autre ne cesse d'inspirer des poéticiens, des artistes et des philosophes à travers le monde.

A doctor by training, Victor Segalen had many other talents. Enlisted in the French Navy, he travelled to Polynesia and China, coming across other civilisations. The notebooks he had filled out during his journeys would provide main material for his later works. While the colonial empires laid their hands on the riches of African and Asian peoples, this poet was enamored with the diversity of the world challenged the folkloric vision of the other; he recovers the word "exoticism" and the "Exote" which he inscribes in a "diametrically opposed problematic" (Manceron, 1991: 14). "The esthetic of the diverse", exposed in his Essay on Exoticism, grants a preponderant role to the "imaginary" and in the possibilities "dream" as fundamental "aptitudes" of the "l'Exote »: A type of traveler endowed with "the power to conceive the other". The richness in this fashion to perceive the other keeps inspiring poets, artists and philosophers throughout the world.

\section{INDEX}

Mots-clés : Segalen (Victor), exotisme, exote, divers, imaginaire

Keywords : Segalen (Victor), exoticism, exote, diverse, imaginary

\section{AUTEUR}

\section{ADEL HABBASSI}

Université de Tunis El Manar

ahabbassi2019[at]gmail.com 